\title{
Paraspinal Muscle Activity Differences in Various Subtypes of Idiopathic Scoliosis
}

\author{
Naveen Kumar Balne ${ }^{1}$, S. Afshan Jabeen ${ }^{2}$, Neeharika Mathukumalli ${ }^{3}$, Sailaja S $^{4}$ \\ ${ }^{1}$ M.P.T Neurology, Faculty Lecturer, Department of Physiotherapy, Nizam's Institute of Medical Sciences \\ ${ }^{2,3}$ M.D. D.M Neurology, Additional Professor, Department of Neurology, Nizam's Institute of Medical Sciences \\ ${ }^{4}$ M.Sc. PGDNT, Senior Neurotechnologist, Department of Neurology, Nizam's Institute of Medical Sciences, \\ Punjagutta, Hyderabad - 500082, Telangana, India.
}

Corresponding Author: Naveen Kumar Balne

\begin{abstract}
Background: Scoliosis refers to deviation of spinal alignment in three dimensional planes. In response to functional demands Muscle fibers change in motor unit function. Knowledge of difference in muscle fiber activity in functional situations allows us to plan curve specific effective physical therapy.

Methodology: All study subjects were categorised based on Rigo Classification and assessed for para spinal muscle activity by Surface Electro Myography at cervical, thoracic, lumbar, concave- convex sides of apex and lumbar /lumbo sacral regions of spine in general sequence and curve specific sequence.

Results: Total number of subjects were 25 (16 female and 9 male). Age ranging from 12 to 39 years and Risser from 1-5. Overall maximum amplitudes recorded in antigravity positions. Amplitudes of as low as $(\mathrm{M} \pm \sigma=$ mean \pm standard deviation) $\mathrm{M} \pm \sigma: 0.92 \pm 0.79 \mu \mathrm{v}$ to $\mathrm{M} \pm \sigma: 1411.6 \pm 734.9 \mu \mathrm{v}$ were found.

Conclusion: Differences in right and left paraspinal activity was observed in general and specific sequences, but this difference was not consistent in general and specific sequence surface electro myography. Maximum amplitudes found at lumbar and lumbo sacral regions on antigravity positions than other positions. Convex side has more activity than concave sides at apex regions. Differences were not similar in all positions, this difference in left and right side was varying with curve type and type of movement. Results of paraspinal activity may be used for planning of suitable exercises after achieving best possible correction in sagittal and frontal planes.
\end{abstract}

Keywords: Paraspinal muscle activity differences, idiopathic scoliosis, deviation of spinal alignment

\section{INTRODUCTION}

Scoliosis refers to deviation of spinal alignment in three dimensions. Scoliotic spine shows lateral deviation and rotation of segments (1). It can be structural and nonstructural (functional). Subjects with structural scoliosis present with morphological changes of the vertebral bodies, where as in functional scoliosis spine appears to have coronal plane deviation which is reversible and structurally normal. The term Idiopathic means "denoting a disease or condition, the cause of which is not known or that arises spontaneously" (2). This is the most common type of scoliosis. Adolescent idiopathic scoliosis is a common disease with an overall prevalence of $0.47-5.2 \%$ (3). Approximately $80 \%$ diagnosed scoliosis cases are idiopathic and remaining 20\% are secondary to another pathological process (4). This condition may not result from lifting and carrying heavy objects, sports, postural deviations or small leg length discrepancies.

A motor unit (term introduced by Sherrington (5) is an alpha motor neuron (originating in the spinal cord) and all of the 
muscle fibers that it innervates. Muscle fibers are classified based on biochemical nature and histochemistry into three types 1 . Fast-twitch glycolytic (FG), 2. Fast-twitch oxidative (FOG), and 3. Slow-twitch oxidative (SO) (6). Each motor neuron concerned with action of a unique group of muscle fibers. All muscle fibers, in each motor unit will have similar characteristics. These motor units are categorized into fast twitch motor units and slow twitch motor units based on contractile speed. Para spinal muscles contain mainly type -1 fibers, and they perform predominantly postural function (7).

There is change in para spinal fibre distribution in scoliosis, Para spinal muscles on both sides of scoliosis curve were examined for morphological and functional status (8). Predominance of type 1 fibers is observed on convex side, in association with functional change (9). In response to functional demands Muscle fibers not only change their morphology and also they convert from one type to other leading to change in motor unit function and category.

Knowledge of difference in muscle fiber activity in functional situations allows us to plan curve specific physical therapy. As muscle fiber morphology and functions are inter related, studies on electrical activity of para spinal muscles in persons with scoliosis, on convex and concave side of the curve is necessary. Knowing Para spinal activity in various types of idiopathic scoliosis is going to help current physiotherapy professional to plan individual specific exercise program. Semg is non-invasive method of recording electrical activity of muscle. Surface electro myography (Semg) was utilized to record the summated electrical activity of Para spinal muscles and find bilateral paraspinal muscle activity imbalances in scoliosis subjects in static and dynamic positions (10).

Objective: This study was aimed at identifying maximum and minimum muscle activity of paraspinal regions on left and right sides in anterior, posterior, left, right sway, prone position and antigravity position by surface electro myography (Semg) in various subcategories (G2, N3N4 G1-2) of adolescent and adult idiopathic structural spinal curve.

\section{METHODOLOGY}

Study was conducted at Nizam's Institute of Medical Sciences, Department of Neurology and Department of Physiotherapy. After ethical clearance and informed consent, Persons who met the inclusion criteria were included in the study. Study population was screened for deviations of spine in coronal and sagittal planes, Persons with deviated spinal curves were categorized as per "Rigo Classification (11)" into G1, G2, N3N4, G1-2 and these curves are further sub categorized into $\mathrm{A} 1, \mathrm{~A} 2, \mathrm{~A} 3, \mathrm{~B} 1, \mathrm{~B} 2, \mathrm{C} 1, \mathrm{C} 2, \mathrm{E} 1$ and E2. All subjects were assessed for SEMG of para spinal muscles of cervical, thoracic, lumbar, concave- convex sides of apex and lumbar /lumbo sacral regions of spine in general sequence and curve specific sequence. Person with any of the exclusion criteria was excluded which affect the spine or its Paraspinal muscles.

\section{Inclusion criteria:}

1. Male and female, from 12 years to 40 years),

2. Subjects diagnosed with Idiopathic scoliosis- (G2, N3N4 \& G 1-2)

3. A spinal curve with at least 10degrees or more.

4. Subjects willing to provide written informed consent.

Exclusion criteria: Subjects with G1 category spine, Post-surgical, Congenital scoliosis, Inflammatory, Neoplastic, metabolic, Infectious, Developmental, Traumatic conditions, Psychological, psychiatric problems, Ehlers - Danlos syndrome, Mesenchymal disorders, Neurofibromatosis and Neuro muscular diseases are excluded. Subjects with any other disorders which lead to changes in spinal curves are excluded. 


\section{Surface electro myography (Semg):}

Subjects were informed about the study procedure. Each participant was familiarised with the body movement before starting the test: anterior sway, posterior sway, left sway, right sway. Semg was performed using Nicolet EDX machine by a senior Neurotechnologist. Initially a general sequence was conducted during the sway movements and followed by curve specific sequence which excludes cervical and includes prone and anti-gravity positions. Amplitudes were recorded and used for study of paraspinal activity.

Semg of paraspinal muscles was recorded both general and curve specific sequence during postural sway movements, prone and antigravity positions using bipolar, $10 \mathrm{~mm}$ diameter pre-gelled $\mathrm{Ag} / \mathrm{AgCl}$ circular electrodes with inter electrode distance 1.5 to $3 \mathrm{~cm}$ was used for noise reduction and to decrease the electrode-skin impedance. Filter settings kept between $20 \mathrm{~Hz}$ to $10 \mathrm{kHz}$. Electrodes were placed in the line of arrangement of fibers (parallel).Electrodes were adhered to skin with adhesive electrodes. Cables were connected to the multichannel physiologic recording device (Figure 1a) or the amplifier box and secured in such a way that desired movements were possible without any restrictions and no pull on the electrodes during movement (Figure 1b: Semg recording).

In general sequence Semg study, all subjects were assessed for their paraspinal activity at cervical, thoracic and lumbar region on left and right sides during all postural sway activities (anterior sway, posterior sway, left bending and right bending). In curve specific sequence Semg study similar subjects were assessed for Semg of paraspinal muscles on lumbo sacral region, concave, convex sides of spine during postural sway movements, prone and antigravity positions. (Figure 2: positions of testing) depending type of curve.

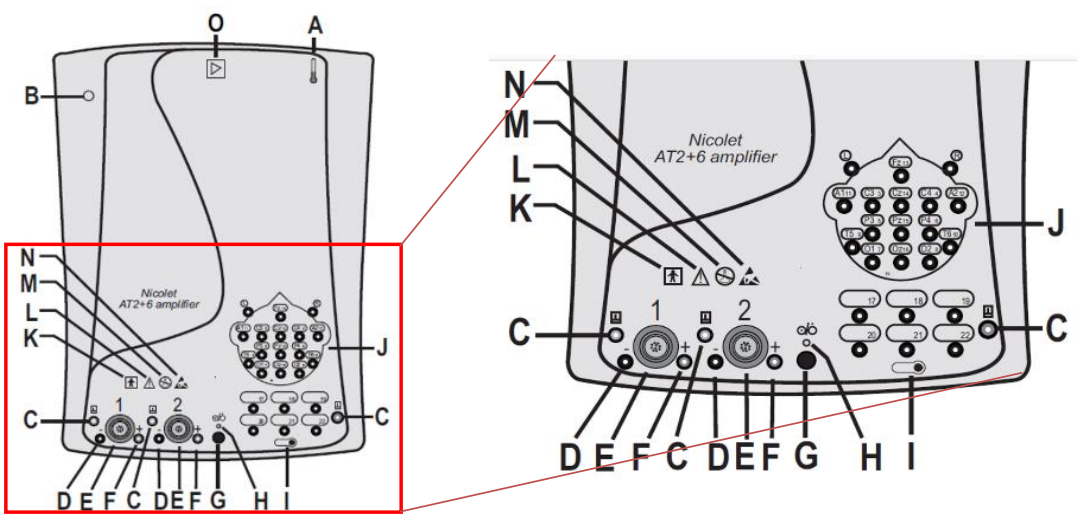

Figure 1a: Multichannel physiologic recording device

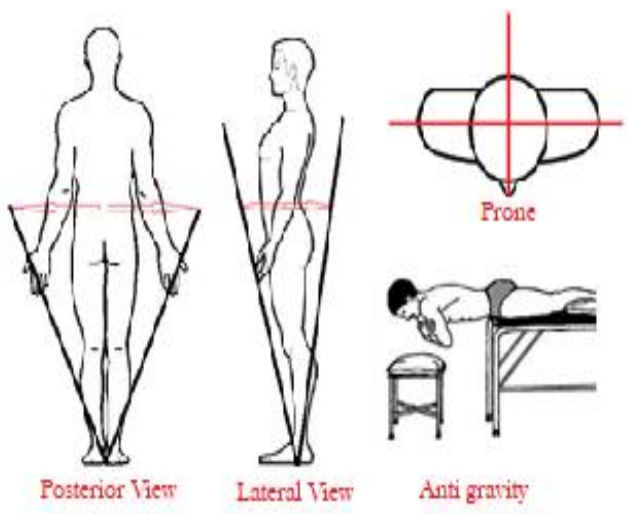

Figure 2 Positions of testing

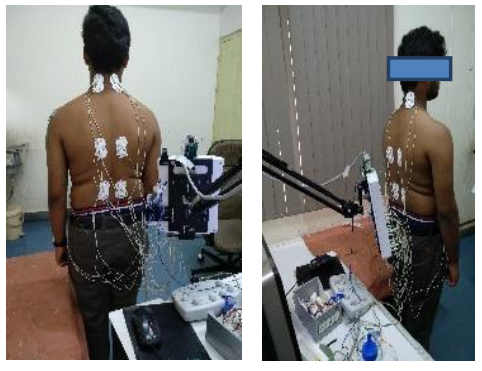

Figure 1b: Semg recording

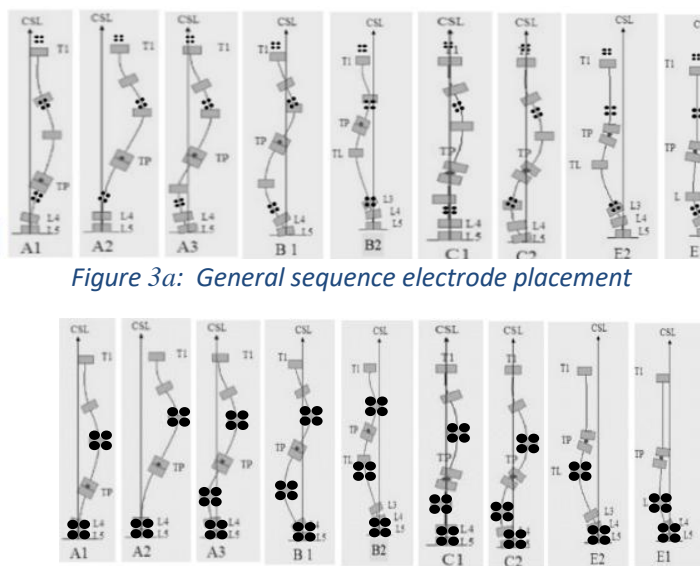

Figure $2 b$ Curve specific sequence electrode placement 
Placement of electrodes was determined by means of palpation. The indifferent electrode was positioned at the right shoulder. In general sequence recording bipolar electrodes were placed on 1. Either sides of cervical (C3-C4), thoracic (T5-T6), and lumbar (L2-L3) Para spinal regions 2. In curve specific sequence electrodes were placed on Convex and concave side of apex of spinal curves and on either sides of L3-S1 (lumbo sacral region). Schema of electrode placement used for Para spinal region shown below (figure $3 \mathrm{a}$ : general sequence, $3 \mathrm{~b}$ : curve specific sequence).

All electrode cables were connected to Multichannel physiologic recording device in the following way (Table 1). Electrode connection in general sequence for subjects of all types of curves was same, Cervical left electrodes to DN1; cervical right electrodes to DN2; thoracic left to 17 and 20; thoracic right to 18 and 21 ; lumbar left to 19 and 22; lumbar right to $\mathrm{L}$ and $\mathrm{R}$. For Curve specific sequence surface Semg recording electrodes were connected based on type of curve as shown below (Table 1).

Table 1 Electrodes connection to Multichannel physiologic recording device

\begin{tabular}{|c|c|c|c|c|c|c|c|c|c|c|c|}
\hline \multirow[t]{2}{*}{ Sequence } & \multirow[t]{2}{*}{ Type } & \multicolumn{2}{|c|}{ Cervical } & \multicolumn{2}{|c|}{ Thoracic } & \multicolumn{2}{|c|}{ Thoraco lumbar } & \multicolumn{2}{|c|}{ Lumbar } & \multicolumn{2}{|c|}{ Lumbo sacral } \\
\hline & & $\mathbf{L t}$ & $\mathbf{R t}$ & $\mathbf{L t}$ & $\mathbf{R t}$ & Lt & $\mathbf{R t}$ & $\mathbf{L t}$ & Rt & Lt & $\mathbf{R t}$ \\
\hline General sequence & All & DN 1 & DN 2 & 17,20 & 18,21 & & & 19,22 & $\mathrm{~L}, \mathrm{R}$ & & \\
\hline \multirow{9}{*}{ 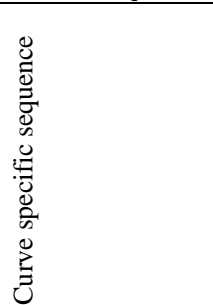 } & A1 & & & DN 1 & DN 2 & & & & & $\mathrm{~L}$ & $\mathrm{R}$ \\
\hline & $\mathrm{A} 2$ & & & DN 1 & DN 2 & & & & & $\mathrm{~L}$ & $\mathrm{R}$ \\
\hline & A3 & & & DN 1 & DN 2 & & & 17,20 & 18,21 & $\mathrm{~L}$ & $\mathrm{R}$ \\
\hline & B1 & & & DN 1 & DN 2 & 17,20 & 18,21 & & & $\mathrm{~L}$ & $\mathrm{R}$ \\
\hline & $\mathrm{B} 2$ & & & DN 1 & $\mathrm{DN} 2$ & 17,20 & 18,21 & & & $\mathrm{~L}$ & $\mathrm{R}$ \\
\hline & $\mathrm{C} 1$ & & & DN 1 & DN 2 & & & 17,20 & 18,21 & $\mathrm{~L}$ & $\mathrm{R}$ \\
\hline & $\mathrm{C} 2$ & & & DN 1 & DN 2 & & & 17,20 & 18,21 & $\mathrm{~L}$ & $\mathrm{R}$ \\
\hline & E1 & & & & & & & 17,20 & 18,21 & $\mathrm{~L}$ & $\mathrm{R}$ \\
\hline & E2 & & & & & 17,20 & 18,21 & & & $\mathrm{~L}$ & $\mathrm{R}$ \\
\hline
\end{tabular}

\section{RESULTS AND DISCUSSION}

Subjects who met were categorised as per Rigo classification and assessed for Semg of paraspinal muscles. Total number of subjects in study were 25, Sample number in each category was 5 subjects in 3C (A1-3,A2-1,A3-1), 4 subjects in 4C(B13,B2-1), 10 subjects in N3N4 (C1-6,C24)and 6 subjects in G1-2(E1-5,E2-1). Gender distribution was 16 female and 9 male. Age ranging from 12 years to 39 years and Risser ranging from 1-5.

Surface Electro Myography (Semg) values of each sub category individual was measured in general sequence and specific sequences, Values were recorded in Micro volts $(\mu \mathrm{v})$. All the data was presented in the form of descriptive statistics, which includes measures of central tendency (Mean and standard deviation) and relevant maximum and minimum values of observed paraspinal Activity was presented. Categories of A2, A3, B2, and E2 were not taken for analysis as they had single samples. All other categories with more than single sample were analysed and presented to show the variations of sequences and right and left sides in different categories of idiopathic scoliosis. Please see demographics in below table 2.

Table 2 Demographic details

\begin{tabular}{|l|l|l|}
\hline & Number & Details \\
\hline Sample & 25 & 16 female, 9 Male \\
\hline Age & 12 to 39 years & mean \pm SD 23.1 \pm 7.1 \\
\hline 3C & 5 & A1-3, A2-1, A3-1 \\
\hline 4C & 4 & B1-3, B2-1 \\
\hline N3N4 & 10 & C1-6,C2-4 \\
\hline G1-2 & 6 & E1-5, E2-1 \\
\hline
\end{tabular}

In Semg of 3C A1category general sequence, maximum amplitude $(\mathrm{M} \pm \sigma$ : 258.4 \pm 185.1 ), was found at right thoracic region during right sway movement and minimum amplitude $(\mathrm{M} \pm \sigma: 43.74 \pm 15.08)$, was found at left thoracic region during anterior sway movement. In all sway movement thoracic and lumbar regions paraspinal muscle activity was more than cervical region. In specific sequence Semg of 3CA1 category, maximum amplitude $(\mathrm{M} \pm \sigma:$ 751 \pm 7.3$)$, was found at right thoracic region in antigravity position, and 
minimum amplitude ( $\mathrm{M} \pm \sigma: 20.7 \pm 17.9)$, was found at left Lumbo sacral region during prone position. During specific sequence Semg convex side paraspinal muscle activity at thoracic and lumbosacral region is more than concave side. There is reduction of convex thoracic region muscle activity during left sway and anterior sway but still activity is higher than concave side thoracic paraspinal muscles.

4CB1 subjects had double curves (thoracic and lumbar), these category curves transition points are located on the concave side of CSVL (central sacral vertical line) and slight imbalance to concave thoracic side. General sequence of this category showed different presentation. There was more paraspinal activity on convex (Rt) thoracic $(\mathrm{M} \pm \sigma:$ 79.9 \pm 73.7$)$ region on posterior sway, (Lt) lumbar region $(\mathrm{M} \pm \sigma$ : 180.2 \pm 72.4$)$ on anterior sway along with increased activity at cervical region $(\mathrm{M} \pm \sigma$ : $116.8 \pm 2.5$ ) on anterior sway. During specific sequence Semg as electrodes were directly placed at curve region and lumbosacral regions, the activation pattern was almost similar but increased level activity was seen at $\mathrm{Lt}$ thoracic $(\mathrm{M} \pm \sigma$ : 126.6 \pm 90.6$)$ than right thoracic region $(\mathrm{M} \pm$ $\sigma: 109.1 \pm 79.4)$, Lt lumbar region $(\mathrm{M} \pm \sigma$ : $528.8 \pm 66)$ showed more activity than $\operatorname{right}(\mathrm{M} \pm \quad \sigma: \quad 455.4 \pm 291.9)$. Lumbosacral region also showed greater activity in antigravity position than other movements. Left lumbosacral region amplitude was $\mathrm{M} \pm$ $\sigma: 517.6 \pm 85.4$ and right side was $\mathrm{M} \pm \sigma$ : 381.3 \pm 163.6 .

C1 category subjects had curves like 4C but transitional pints and $\mathrm{C} 7$ are located on CSVL and had balanced curves with less lumbar curves. During general sequence Semg, maximum amplitude seen at left cervical $(\mathrm{M} \pm \sigma: 87 \pm 21.7)$ and left lumbar $(\mathrm{M} \pm \sigma: 189.7 \pm 177)$ regions on anterior sway, right thoracic $(\mathrm{M} \pm \sigma: 94.9 \pm 81.3)$ on left sway. During specific sequence Semg spinal activity was maximum in antigravity position. Right thoracic $(\mathrm{M} \pm \sigma$ : 394.1 $\pm 3.6 .3)$, Left lumbar $(\mathrm{M} \pm \sigma$ : 1411.6 \pm 734.9$)$ and left lumbo sacral $(\mathrm{M} \pm \sigma$ :
429.2 \pm 244$)$ regions had maximum spinal activity in antigravity position.

C2 category subjects had curves at thoracic and lumbar region with balanced spine and transition point location on CSVL. Subjects with C2 curves had maximum spinal activity at left cervical $(\mathrm{M} \pm \sigma: 0.57 \pm 0.63)$, right lumbar regions $(\mathrm{M} \pm \sigma: 1.31 \pm 1)$ in anterior sway movements and Rt thoracic region $(\mathrm{M} \pm \sigma: 0.92 \pm 0.79)$ in right sway motion. Semg on specific sequence was showed maximum paraspinal activity on antigravity position. Right thoracic $(\mathrm{M} \pm \sigma$ 117.8 \pm 189.2$)$, left lumbar $(\mathrm{M} \pm \sigma: 492.4 \pm 205.6)$ and left lumbo sacral $(\mathrm{M} \pm \sigma: 809.4 \pm 378.8)$ regions are more active than other regions in antigravity position than any other movements.

G1-2 category spine subjects had lumbar region curves, with or without virtual thoracic curves (functional curves), E1 subjects had lumbar apex and E2 category subjects had Thoraco lumbar apex, transitional point was offset to convex lumbar curve side on CSVL. On general sequence Semg left cervical region showed much activity than right during anterior, left and right sway maximum was seen at right sway $(\mathrm{M} \pm \sigma: \quad 75.8 \pm 30.1)$. Left thoracic region had better activity than right side with predominant activity during initiation of posterior sway $(\mathrm{M} \pm \sigma: 157.3 \pm 111.6)$, right lumbar region showed maximum amplitude during anterior sway $(\mathrm{M} \pm \sigma$ : $215.9 \pm 104.2)$ and less activity on posterior sway $(\mathrm{M} \pm \sigma$ : 40.6 \pm 13.9 ), left lumbar region showed maximum activity during anterior sway and less activity on posterior sway. On left sway right lumbar showed more activity $(\mathrm{M} \pm \sigma$ : 133.3 \pm 45.3$)$ less on left side $(\mathrm{M} \pm \sigma$ :

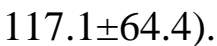

There were studies done in sitting and standing, EMG ratios were studied in apex, upper and lower end vertebra locations. Ratios more than 1 at concave and convex regions correlated with progressive curves in children but in adults the relation between Semg parameters and curve development and progression is another area to be addressed. Paraspinal muscles activity 
is considered as one of the influencing factor in curve development and also in progression (12). Another study was conducted using tilt surface to study paraspinal activity during perturbations towards left side and right side (13). This study was focused on cervical, thoracic and lumbar region paraspinal activity in general sequence and convex, concave sides of spine along with lumbosacral region in curve specific sequence Semg. Results showed differences in right and left side paraspinal amplitudes in general and specific sequences. In general sequence most of the curve types showed better amplitudes during anterior sway at $\mathrm{Lt}$ cervical, Right thoracic and Left lumbar and Lumbo sacral regions. There is coupling of left lumbar and lumbo sacral region Semg activity. In specific sequence the activity of paraspinal muscles is much evident on convex sides of curve in almost all categories. Some curves like G1-2 category and ipsilateral movements showed more left thoracic amplitude than right thoracic region even though apex was on right side. This kind of presentation may be because of compensatory curves or functional type curve seen at thoracic region, or it may be early phase of activation of fast fibers in early stage of movement initiation. Maximum level of amplitude was observed in antigravity positions of curves in all regions. Lumbar and lumbosacral regions were active more than any other region of paraspinal muscles. Differences between convex and concave side paraspinal activity was clearly seen in specific sequence (14) where the electrodes are placed on convex and concave side of the curves.

\section{CONCLUSION}

Differences in right and left paraspinal activity was observed in general and specific sequences, maximum amplitudes found at lumbar and lumbo sacral regions on antigravity position. Differences were not similar in all positions, this difference in left and right side was varying with curve type and type of movement. Results of paraspinal activity may be used for planning of suitable exercises after achieving best possible correction in sagittal and frontal planes. This study guides us to choose suitable strategy of exercises for elongation, and derotation of spine by targeting paraspinal muscles either to facilitate or inhibit with suitable strategies. Study of paraspinal muscles activity by placing electrodes at common locations like cervical, thoracic and lumbar regions in general sequence and concave and convex locations of curve on left and right sides in specific sequence will give us overall paraspinal activity on left and right side. The positions chosen for recording of paraspinal activity are most commonly used positions in our daily activities, hence understanding of results gives insights for physiotherapy professionals for planning of individual therapeutic regimen. Studies may be required to study para spinal muscle activities in A2, A3, B2 and E2 categories. Larger sample studies with comparison of normal individual paraspinal activity might further help in standardising procedure to generalise the results.

\section{Acknowledgement: None}

\section{Conflict of Interest: None}

\section{Source of Funding: None}

\section{Ethical Approval: Approved}

\section{REFERENCES}

1. Chair Larry Lenke MGo3DCTC. Scoliosis research society. [Online].; 2000 [cited 2018 February 22.

2. Concise Medical Dictionary (8ed.). 8th ed.: Oxford University Press; 2010.

3. Markus Rafael Konieczny HSaRK. Epidemiology of adolescent idiopathic scoliosis. Journal of Children Orthopaedics. 2013 Feb; 7(1): p. 3-9.

4. Negrini, S., Donzelli, S., Aulisa, A.G. et al. 2016 SOSORT guidelines: orthopaedic and rehabilitation treatment of idiopathic scoliosis during growth. Scoliosis 13, 3 
(2018). https://doi.org/10.1186/s13013-0170145-8.

5. Burke RE. Revisiting the Notion of 'motor unit types. In Binder MD, editor. 'Peripheral and Spinal Mechanisms in the Neural Control of Movement. 12334821999th ed.; 1999. p. 167-175.

6. Wayne Scott JSSAB. Human Skeletal Muscle Fiber Type Classifications. Physical Therapy. 2001 November 1; 81(11): p. 1810-1816.

7. A. F. Mannion et. al. Muscle fibre size and type distribution in thoracic and lumbar regions of erector spinae in healthy subjects without low back pain: normal values and sex differences. J Anat. 1997 May;190 ( Pt 4)(Pt 4):505-13. doi: 10.1046/j.14697580.1997.19040505.x.

8. I.Stetkarovaa et al. Neurophysiology and biopsy of paraspinal muscles in idiopathic scoliosis. Clinical Neurophysiology. 2016, March; 127(3): p. e105.

9. Herbert MA BW. Paraspinal muscle stimulation for the treatment of idiopathic scoliosis in children. Orthopedics. 1987 August; 10(8): p. 1125-32.

10. JD Cassidy et al. The role of paraspinal muscles in the pathogenesis of idiopathic scoliosis: A preliminary EMG study.
Journal of Canadian chiropractic association. 1987 December; 31(4).

11. Manuel D Rigo et al. A specific scoliosis classification correlating with brace treatment: description and reliability. Scoliosis and Spinal Disorders. 2010 January 27 .

12. John Cheung et al. A preliminary study on electromyographic analysis of the paraspinal musculature in idiopathic scoliosis. Europian spine journal. 2005 March; 14(2).

13. Claude Perret JR. Electromyographic responses of paraspinal muscles to postural disturbance with special reference to scoliotic children. Journal of Manipulative and physiological therapeutics. 2004 JulyAugust.

14. Gnahoua Zoabli et al. Back muscles biometry in adolescent idiopathic scoliosis. The spine journal. 2007 May -June; 7(3): p. 338-44.

How to cite this article: Balne BK, S. Afshan Jabeen, Mathukumalli $\mathrm{N}$ et.al. Paraspinal muscle activity differences in various subtypes of idiopathic scoliosis. Int J Health Sci Res. 2021; 11(9): 113-119. DOI: https://doi.org/10. 52403/ijhsr.20210917 